\title{
A Radiation-Hydrodynamical Model for Supermassive Black Hole-to-Bulge Mass Relation and Quasar Formation
}

\author{
Masayuki Umemura \\ umemura@rccp.tsukuba.ac.jp \\ Center for Computational Physics, University of Tsukuba, Tsukuba, Ibaraki 305, Japan
}

To appear in Astrophysical Journal Letters

\begin{abstract}
As a potential mechanism to build up supermassive black holes (BHs) in a spheroidal system, we consider the radiation drag effect by bulge stars, which extracts angular momentum from interstellar gas and thus allows the gas to accrete onto the galactic center. With incorporating radiation hydrodynamical equation with simple stellar evolution, it is shown that the BH-tobulge mass ratio, $f_{\mathrm{BH}}$, is basically determined by a fundamental constant, that is, the energy conversion efficiency for nuclear fusion of hydrogen to helium, $\varepsilon=0.007$. More specifically, $f_{\mathrm{BH}}$ is predicted to be $0.3 \varepsilon-0.5 \varepsilon$. Based on the present model for $\mathrm{BH}$ growth, a scenario for quasar formation is addressed in relation to ultraluminous infrared galaxies.
\end{abstract}

Subject headings: black hole physics — accretion — galaxies: active — galaxies: formation — galaxies: evolution — galaxies: nuclei — galaxies: starburst

\section{Introduction}

The recent compilation of the kinematical data for galactic centers in both inactive and active galaxies has revealed that the estimated mass of a central 'massive dark object' (MDO), which is the nomenclature for a supermassive $\mathrm{BH}$ candidate, does correlate with the properties of galactic bulges. The demography of MDOs has shown a number of intriguing relations: 1) The $\mathrm{BH}$ mass exhibits a linear relation to the bulge mass with the ratio of $f_{\mathrm{BH}} \equiv M_{\mathrm{BH}} / M_{\text {bulge }}=0.001-0.006$ as a median value (Kormendy \& Richstone 1995; Magorrian et al. 1998; Richstone et al. 1998; Gebhardt et al. 2000; Ferrarese \& Merritt 2000; Merritt \& Ferrarese 2001a) (It is noted that the bulge means a whole galaxy for an elliptical galaxy.) 2) The BH mass correlates with the velocity dispersion of bulge stars with a power-law relation as $M_{\mathrm{BH}} \propto \sigma^{n}, n=3.75$ (Gebhardt et al. 2000a) or 4.72 (Ferrarese \& Merritt 2000; Merritt \& Ferrarese 2001a, 2001b). 3) The ratio $f_{\mathrm{BH}}$ tends to grow with the age of youngest stars in a bulge until $10^{9} \mathrm{yr}$ (Merrifield et al. 2000). 4) In disk galaxies,
$f_{\mathrm{BH}}$ is significantly smaller than 0.001 when the disk stars are included (Salucci et al. 2000; Sarzi et al. 2001). 5) For quasars, $f_{\mathrm{BH}}$ is level with that for elliptical galaxies (Laor 1998). 6) As for Seyfert galaxies, the mass ratio is under controversy. The estimation by the reverberation mapping suggests that $f_{\mathrm{BH}}$ in Seyfert 1 galaxies is considerably smaller than 0.001 (Wandel 1999; Gebhardt et al. 2000b), although the reverberation mapping method may cause a systematic underestimate of BH mass (Krolik 2001). However, the measurements of $\mathrm{H}_{\beta}$ line widths give $f_{\mathrm{BH}} \simeq 0.0025$ for Seyfert 1's as well as quasars (McLure \& Dunlop 2001). On the other hand, the BH mass-tovelocity dispersion relation in Seyfert 1 galaxies seems to hold good in a similar way to elliptical galaxies (Nelson 2000; Gebhardt et al. 2000b). All of these correlations imply that the formation of a supermassive BH is likely to be physically linked to the formation of a galactic bulge which harbors a supermassive $\mathrm{BH}$. In addition, a great deal of recent efforts have revealed that quasar host galaxies are mostly luminous and well evolved early- 
type galaxies (McLeod \& Rieke 1995; Bahcall et al. 1997; Hooper, Impey, \& Foltz 1997; McLeod, Rieke, \& Storrie-Lombardi 1999; Brotherton et al. 1999; Kirhakos et al. 1999; McLure et al. 1999; McLure, Dunlop, \& Kukula 2000). These findings, combined with the $\mathrm{BH}$-to-bulge relations, suggest that the formation of a supermassive $\mathrm{BH}$, a bulge, and a quasar is mutually related.

Some theoretical models have been considered to explain the BH-to-bulge correlations, e.g., hydrodynamical ones including a wind-regulation model (Silk \& Rees 1998) or an inside-out accretion model (Adams, Graff, \& Richstone 2001), and self-interacting dark matter model (Ostriker 2000). But, little has been elucidated regarding the physics on the angular momentum transfer which is inevitable for $\mathrm{BH}$ formation, since the rotation barrier by the tidal spin up in a growing density fluctuation is given by

$$
\frac{R_{\text {barr }}}{R_{\text {Sch }}} \approx 10^{6}\left(\frac{M_{b}}{10^{10} M_{\odot}}\right)^{-2 / 3}\left(\frac{\lambda}{0.05}\right)^{2}(1+z)^{-1}
$$

in units of the Schwarzshild radius $R_{\mathrm{Sch}}$, where $M_{b}$ is the baryonic mass, $z$ is the cosmological redshift, and $\lambda$ is the spin parameter which provides the ratio of circular velocity to velocity dispersion of dark matter (Sasaki \& Umemura 1996). Here, $R_{\text {barr }}$ is estimated by $R_{b a r r}=j_{b}(z)^{2} / G M_{b}$, where $j_{b}$ is the specific angular momentum given by $j_{b} \simeq R_{\max } \sigma \lambda$ with $R_{\max }$ being the maximum expansion radius of the fluctuation. Furthermore, required mechanisms for $\mathrm{BH}$ formation must work effectively in a spheroidal system like a bulge. The $\alpha$-viscosity or non-axisymmetric gravitational instabilities would effectively transfer angular momentum once a disk-like system forms, but they are not likely to work in a spheroidal system. In this Letter, as a potential mechanism in a spheroidal system, the relativistic drag force by the radiation from bulge stars is considered, and the $\mathrm{BH}$-to-bulge ratio is derived with incorporating radiation hydrodynamics jointly with simple stellar evolution in a bulge. As a result, the $\mathrm{BH}$-to-bulge ratio is basically determined by the energy conversion efficiency for nuclear fusion of hydrogen to helium, $\varepsilon=0.007$. Also, in relation to $\mathrm{BH}$ growth, a scenario for quasar formation is addressed.

\section{Radiation Drag-Induced Mass Accre- tion}

We suppose a simple two-component system which consists of a spherical stellar bulge and dusty gas within it. The exact expressions for the radiation drag are found in some literatures (Umemura, Fukue, \& Mineshige 1997; Fukue, Umemura, \& Mineshige 1997). For the total luminosity $L_{*}$ of a uniform bulge, the radiation energy density is given by $E \simeq L_{*} / c R^{2}$, where $c$ is the light speed and $R$ is the radius of the bulge. Then, the angular momentum loss rate by the radiation drag is given by $d \ln J / d t \simeq-\chi E / c$, where $J$ is the total angular momentum of gaseous component and $\chi$ is the mass extinction coefficient which is given by $\chi=\kappa / \rho$ with dust absorption coefficient $\kappa$ and gas density $\rho$. Therefore, in an opticallythin regime, $d \ln J / d t \simeq-\tau L_{*} / c^{2} M_{g}$, where $\tau$ is the total optical depth of the system and $M_{g}$ is the total mass of gas. In an optically-thick regime, the radiation drag efficiency is saturated due to the conservation of the photon number (Tsuribe \& Umemura 1997). Thus, an expression of the angular momentum loss rate which includes both regimes is given by $d \ln J / d t \simeq-\left(L_{*} / c^{2} M_{g}\right)(1-$ $\left.\mathrm{e}^{-\tau}\right)$. In practice, it is likely that optically-thin surface layers are stripped from optically-thick clumpy clouds by the radiation drag, and the stripped gas losing angular momentum accretes onto the center (Kawakatu \& Umemura 2001). Since the radiative cooling is effective in the surface layers, the accretion is likely to proceed in an isothermal fashion until an optically-thick massive dark object forms. Then, the mass accretion rate is estimated to be

$$
\dot{M}=-M_{g} \frac{d \ln J}{d t} \simeq \frac{L_{*}}{c^{2}}\left(1-\mathrm{e}^{-\tau}\right) .
$$

In an optically-thick regime, this gives simply $\dot{M}=L_{*} / c^{2}$, which is numerically $\dot{M}=$ $0.1 M_{\odot} \mathrm{yr}^{-1}\left(L_{*} / 10^{12} L_{\odot}\right)$. This rate is comparable to the Eddington mass accretion rate for a black hole with $10^{8} M_{\odot}$, that is, $\dot{M}_{\text {Edd }}=$ $0.2 M_{\odot} \mathrm{yr}^{-1} \eta^{-1}\left(M_{\mathrm{BH}} / 10^{8} M_{\odot}\right)$, where $\eta$ is the energy conversion efficiency. For the moment, $\eta=0.42$ for an extreme Kerr black hole is assumed. For the moment, an optically-thick stage is considered. Then, the mass of an MDO is esti- 
mated by

$$
M_{\mathrm{MDO}}=\int_{0}^{t} \dot{M} d t \simeq \int_{0}^{t} L_{*} / c^{2} d t .
$$

The next task is to construct a model for bulge evolution. Here, we employ a simplest analytic model. The gas fraction $f_{g} \equiv M_{g} / M_{b}$ of the system is regulated by

$$
\frac{d f_{g}}{d t}=-S(t)+F(t)
$$

where $S(t)$ is the star formation rate which is assumed to be a Schmidt law, $k f_{g}$, and $F(t)$ is the mass loss rate from stars which includes supernova explosions and stellar winds. If we invoke the instantaneous recycling approximation, then $F(t)=S(t)(1-\alpha)$, where $\alpha$ is the net efficiency of the conversion into stars after subtracting the mass loss. Then, we have an analytic solution as $f_{g}=\mathrm{e}^{-\alpha k t}$. Hence, the star formation rate is $\dot{M}_{*} / M_{b}=S(t)=k \mathrm{e}^{-\alpha k t}$. The radiation energy emitted by a main sequence star over its lifetime is $0.14 \varepsilon$ to the rest mass energy, where $\varepsilon$ is the energy conversion efficiency of nuclear fusion from hydrogen to helium, which is 0.007 . Thus, the luminosity of the bulge is estimated to be $L_{*}=0.14 \varepsilon k \mathrm{e}^{-\alpha k t} M_{b} c^{2}$. By substituting this in (2), we find

$$
M_{\mathrm{MDO}}=0.14 \varepsilon \alpha^{-1} M_{b}\left(1-\mathrm{e}^{-\alpha k t}\right) .
$$

The term $M_{b}\left(1-\mathrm{e}^{-\alpha k t}\right)$ represents just the stellar mass in the system, that is, $M_{\text {bulge }}$ observationally. As a result, the MDO mass to bulge mass ratio is given by

$$
M_{\mathrm{MDO}} / M_{\mathrm{bulge}}=0.3 \varepsilon \alpha_{0.5}^{-1}
$$

where $\alpha_{0.5}=\alpha / 0.5$. It should be noted that the ratio is time-independent and also regardless of the star formation rate $k$. This result implies that the ratio is basically determined by the total number of emitted photons.

\section{BH Growth and Quasar Formation}

From an observational point of view, the mass of an $\mathrm{MDO}$ is often regarded as $\mathrm{BH}$ mass. But, in the present model they should be distinguished from each other if one strictly defines the $\mathrm{BH}$ mass by the mass inside the event horizon. In the present radiation drag model, it is not very likely that all the angular momentum of stripped gas is removed thoroughly. Hence, the MDO presumably forms a massive and compact rotating disk due to a little residual angular momentum. Then, the $\mathrm{BH}$ is more likely to grow via accretion in the MDO, rather than the prompt collapse of the MDO. If the mass accretion within the MDO is driven by the viscosity, the AGN activity may be ignited. Supposing the mass accretion onto the $\mathrm{BH}$ horizon is limited by an order of the Eddington rate, the $\mathrm{BH}$ mass grows according to

$$
M_{\mathrm{BH}}=M_{0} \mathrm{e}^{\nu t / t_{\mathrm{Edd}}},
$$

where $\nu$ is the ratio of the $\mathrm{BH}$ accretion rate to the Eddington rate, $\nu=\dot{M}_{\mathrm{BH}} / \dot{M}_{\mathrm{Edd}}$, and $t_{\mathrm{Edd}}$ is the Eddington time-scale, $t_{\mathrm{Edd}}=1.9 \times 10^{8} \mathrm{yr}$. Here $M_{0}$ is the mass of a seed $\mathrm{BH}$, which could be a remnant of a massive population III star with $10^{2-3} M_{\odot}$ (Carr, Bond, \& Arnett 1984; Nakamura \& Umemura 2001), or an early formed massive BH with $\sim 10^{5} M_{\odot}$ (Umemura, Loeb, \& Turner 1993). When the $\mathrm{BH}$ growth is given by (6), it is delayed from the growth of the MDO given by (4). In Figure 1, this delay is schematically shown. The BH mass reaches $M_{\mathrm{MDO}}$ at a time $t_{\text {cross }}$ when (6) equals (4). We can calculate $t_{\text {cross }}$ to find $t_{\text {cross }} \approx 10 \nu^{-1} t_{\mathrm{Edd}} \approx 10^{9} \nu^{-1} \mathrm{yr}$, although it has weak dependence on $k$. As seen in Figure 1, during $t<t_{\text {cross }}$, the $\mathrm{BH}$ mass fraction $f_{\mathrm{BH}}$ increases with time and therefore possibly increases with the metallicity of the system. At $t>t_{\text {cross }}$, almost all of the MDO matter has fallen onto the central $\mathrm{BH}$, and therefore the $\mathrm{BH}$ mass fraction is settled to $f_{\mathrm{BH}}=M_{\mathrm{MDO}} / M_{\text {bulge }}=0.3 \varepsilon \alpha_{0.5}^{-1}=0.002 \alpha_{0.5}^{-1}$.

So far, the optically-thick regime has been assumed. However, before $t_{\text {cross }}$ the interstellar gas may be blown out by a galactic wind and the system could be transparent. Recently, it is argued that the color-magnitude relation of bulges can be reproduced if a galactic wind sweeps away the gas at the epoch of a few $10^{8} \mathrm{yr}$ (Kodama \& Arimoto 1997). If the system becomes optically-thin at a wind epoch $t_{\mathrm{w}}$, the radiation drag-induced mass accretion practically stops owing to the reduced efficiency of radiation drag due to the small optical depth. Then, the final MDO mass is settled to $M_{\mathrm{MDO}}=0.14 \varepsilon \alpha^{-1} M_{b}\left(1-\mathrm{e}^{-\alpha k t_{\mathrm{w}}}\right)$. If the timescale of star formation $(\alpha k)^{-1}$ is longer than $t_{\mathrm{w}}$, the final MDO mass can be reduced. 
But, $M_{\mathrm{MDO}} / M_{\text {bulge }}$ is time-independent as stated above. When all gas of the MDO eventually falls onto the $\mathrm{BH}$, again $f_{\mathrm{BH}}=0.3 \varepsilon \alpha_{0.5}^{-1}$ is achieved at $t_{\text {cross }}$.

Based on the present model for $\mathrm{BH}$ growth, a picture of quasar formation may be constructed as shown schematically in Figure 1. Recently, the Eddington ratio is estimated to be $\nu \approx 0.1$ for quasars (McLeod, Rieke, \& Storrie-Lombardi 1999). Hence, $t_{\text {cross }} \approx 10^{10} \mathrm{yr}$ and therefore $t_{\text {cross }} \gg t_{\mathrm{w}}$. Since the bulge luminosity decreases as $L_{*} \propto \mathrm{e}^{-\alpha k t}$, the stage at $t<t_{\mathrm{w}}$ is a bright, optically-thick phase, which may correspond to a ultraluminous infrared galaxy (ULIRG). Then, a ULIRG $\left(t<t_{\mathrm{w}}\right)$ harbors a more or less active nucleus. This can be regarded as a model for a paradigm of the evolution of ULIRGs to quasars proposed by Sanders et al. (1998) and Norman \& Scoville (1998). Even at $t>t_{\mathrm{w}}, M_{\mathrm{BH}}$ still continues to grow until $t_{\text {cross }}$, and therefore the AGN brightens with time if the Eddington ratio is constant. This optically-thin phase may correspond to quasar phenomena. Its early phase might be observed as a narrow line type 1 AGN (Mathur 2001). After the AGN luminosity ( $\left.L_{\mathrm{AGN}}\right)$ exhibits a peak at $t_{\text {cross }}$, it fades out abruptly, not only because the mass accretion rate drops promptly in the optically-thin phase, but also because the energy conversion efficiency is in proportion to $\dot{M} / \dot{M}_{\text {Edd }}$ for an optically-thin advectiondominated accretion flow (e.g. Kato, Fukue, \& Mineshige 1998). The later fading nucleus could be a low luminosity AGN (LLAGN) (e.g. Kawaguchi \& Aoki 2001). As a consequence, $f_{\mathrm{BH}}$ increases with increasing $L_{\mathrm{AGN}}$ up to $f_{\mathrm{BH}}=0.3 \varepsilon \alpha_{0.5}^{-1}$. Then, $f_{\mathrm{BH}}$ for quasars is predicted to be similar to that of ellipticals, although possibly a bit smaller. As for $f_{\mathrm{BH}}$ for Seyfert 1 galaxies, naively it is expected to be level with that for quasars, but circumnuclear starbursts might predominantly regulate the mass accretion (Umemura, Fukue, \& Mineshige 1998).

Finally, we point out that further mass accretion could be induced also by the AGN luminosity. The mass accumulated by this self-induced accretion is maximally $M_{\text {Ind }}=\eta(1-\eta)^{-1} M_{\mathrm{BH}}$, if the successive induction is included. Hence, at later stages as $t>t_{\text {cross }}$, we have $f_{\mathrm{BH}}=$ $\left(M_{\mathrm{MDO}}+M_{\text {Ind }}\right) / M_{\text {bulge }}$. Thus, the BH-to-bulge

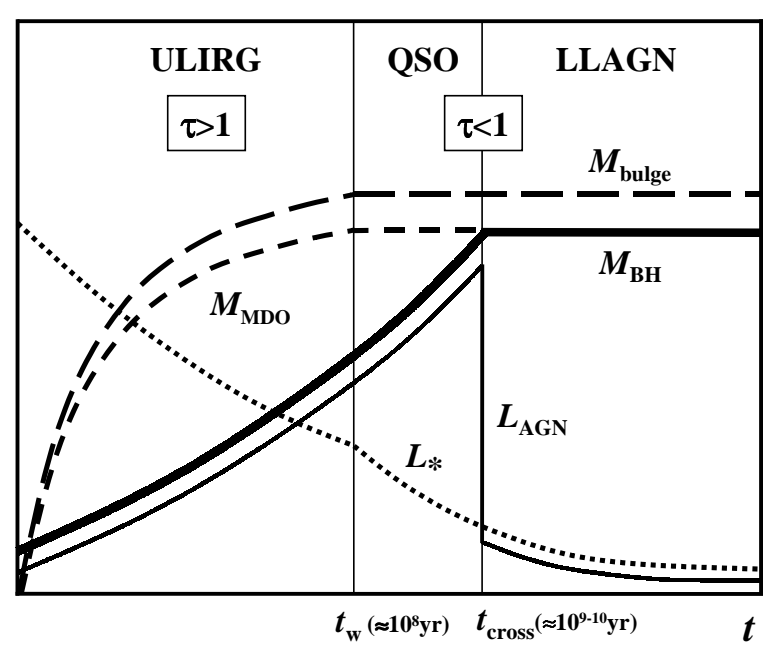

Fig. 1.- A schematic sketch of black hole growth and a scenario for quasar formation. The abscissa is time and the ordinate is mass or luminosity in arbitrary units. $M_{\text {bulge }}$ is the mass of stellar component in the bulge. $M_{\mathrm{MDO}}$ is the mass of the massive dark object (MDO). $M_{\mathrm{BH}}$ is the mass of the supermassive $\mathrm{BH} . L_{*}$ and $L_{\mathrm{AGN}}$ are the bulge luminosity and the black hole accretion luminosity, respectively. $t_{\mathrm{w}}$ is the galactic wind timescale, and $t_{\text {cross }}$ is defined so that $M_{\mathrm{MDO}}=M_{\mathrm{BH}}$. 
mass ratio could become

$$
f_{\mathrm{BH}}=0.14 \varepsilon \alpha^{-1}(1-\eta)^{-1}=0.5 \varepsilon \alpha_{0.5}^{-1},
$$

if an extreme Kerr $\mathrm{BH}(\eta=0.42)$ is assumed, whereas the self-induction factor $(1-\eta)^{-1}=1.06$ for a Schwartzschild BH $(\eta=0.057)$ brings no substantial increase on $f_{\mathrm{BH}}$. In the long run, although leaving some uncertainties with evolution model including $\alpha_{0.5}, f_{\mathrm{BH}}$ is predicted to be $0.3 \varepsilon-0.5 \varepsilon$.

\section{Discussion}

The radiation drag efficiency could be strongly subject to the effect of geometrical dilution (Umemura, Fukue, \& Mineshige 1998; Ohsuga et al. 1999). If the system is spherical, the emitted photons are effectively consumed within the system, whereas a large fraction of photons can escape from a disk-like system and thus the drag efficiency tends to be considerably reduced. Although this is very qualitative, the geometrical dilution may be the reason why $f_{\mathrm{BH}}$ is observed to be significantly smaller than 0.001 in disk galaxies. For the same reason, $f_{\mathrm{BH}}$ could be lower for a flattened bulge.

Finally, the present mechanism may provide a physical basis for the state-of-the-art cosmological scenarios for the joint formation of galaxies and quasars (Haehnelt \& Rees 1993; Haiman \& Loeb 1998; Kauffmann \& Haehnelt 2000; Monaco, Salucci, \& Danese 2000; Granato et al. 2001; Hosokawa et al. 2001). In cosmological studies, $f_{\mathrm{BH}}$ has been hitherto parameterized so that it should match to the observed $\mathrm{BH}$-to-bulge mass relation. Also, Monaco, Salucci, \& Danese (2000) argue that, in order to account for the observed statistics of quasars and elliptical galaxies, there should be a time delay between the beginning of the star formation and the quasar bright phase. In Granato et al. (2001), the formation of early-type protogalaxies as well as quasars is successfully accounted for if it is assumed that the relationship between the $\mathrm{BH}$ mass and the host mass has been imprinted during the early phase of the quasar and host evolution. In the present paper, the BH-tobulge mass relation is a function of time, but it has been found that in a quasar phase their assumption holds good. In the light of these points, the basic assumptions and results by previous cosmological scenarios for quasar formation are mostly consistent with the present model for quasar formation based on the $\mathrm{BH}$ growth.

The author thanks A. Ferrara, N. Kawakatu, S. Mineshige, T. Nakamoto, K. Ohsuga, and H. Susa for fruitful discussions. Also, the author is indebted to the support by Center for Computational Physics, University of Tsukuba. This work was supported in part by the Grant-in-Aid of the JSPS, 11640225. 


\section{REFERENCES}

Adams, F. C., Graff, D. S., \& Richstone, D. O. 2001, ApJ, 551, L31

Bahcall, J. N., et al. 1997, ApJ, 479, 642

Brotherton, M. S., et al. 1999, ApJ, 520, L87

Carr, B. J., Bond, J. R., \& Arnett, W. D. 1984, ApJ, 277, 445

Ferrarese, L., \& Merritt, D. 2000, ApJ, 539, L9

Fukue, J., Umemura, M., \& Mineshige, S. 1997, PASJ, 49, 673

Gebhardt, K., et al. 2000a, ApJ, 539, L13

- 2000b, ApJ, 543, L5

Granato, G. L., Silva, L., Monaco, P., Panuzzo, P., Salucci, P., Zotti, G. De, \& Danse, L. 2001, MNRAS, 324, 757

Haehnelt, M. G., \& Rees, M. J. 1993, MNRAS, 263,168

Haiman, Z. \& Loeb, A. 1998, ApJ, 503, 505

Hooper, E. J., Impey, C. D., \& Foltz, C. B. 1997, ApJ, 480, L95

Hosokawa, T., Mineshige, S., Kawaguchi, T. Yoshikawa, K. \& Umemura, M. 2001, PASJ, in press

Kato, S., Fukue, J., \& Mineshige, S. 1998, BlackHole Accretion Disk (Kyoto: Kyoto Univ. Press)

Kauffmann, G., \& Haehnelt, M. 2000, MNRAS, 311,576

Kawaguchi, T., \& Aoki, K. 2001, preprint

Kawakatu, N., \& Umemura, M. 2001, MNRAS, submitted.

Kirhakos, S., Bahcall, J. N., Schneider, D. P., \& Kristian, J. 1999, ApJ, 520, 67

Kodama, T., \& Arimoto, N. 1997, A\&A, 320, 41

Kormendy, J., \& Richstone, D. 1995, ARA\&A, 33, 581

Krolik, J. H. 2001, ApJ, 551, 72

Laor, A. 1998, ApJ, 505, L83

2001, ApJ, 553, 677

Magorrian, J., et al. 1998, AJ, 115, 2285

Mathur, S. 2001, AJ, in press (astro-ph/0107163)

McLeod, K. K., \& Rieke, G. H. 1995, ApJ, 454, L77

McLeod, K. K., Rieke, G. H. \& Storrie-Lombardi, L. J. 1999, ApJ, 511, L67
McLure, R. J., Dunlop, J. S., \& Kukula, M. J. 2000, MNRAS, 318, 693

McLure, R. J., Kukula, M. J., Dunlop, J. S., Baum, S. A., O'Dea, C. P., \& Hughes, D. H. 1999, MNRAS, 308, 377

McLure, R. J., \& Dunlop, J. S. 2001, MNRAS, in press (astro-ph/0009406)

Merrifield, M. R., Forbes, Duncan A., \& Terlevich, A. I. 2000, MNRAS, 313, L29

Merritt, D., \& Ferrarese, L. 2001a, MNRAS, 320, L30

- 2001b, ApJ, 547, 140

Mineshige, S., Tsuribe, T., \& Umemura, M. 1998, PASJ, 50, 233

Monaco, P., Salucci, P., \& Danese, L. 2000, MNRAS, 311, 279

Nakamura, F., \& Umemura, M. 2001, ApJ, 548, 19

Nelson, C. H. 2000, ApJ, 544, L91

Norman, C. \& Scoville, N. 1988, ApJ, 332, 124

Ostriker, J. P. 2000, Phys. Rev. Lett., 84, 5258

Ohsuga, K., \& Umemura, M. 2001, ApJ, in press (astro-ph/0105474)

Ohsuga, K., Umemura, M., Fukue, J., \& Mineshige, S. 1999, PASJ, 51, 345

Rees, M. 1984, ARA\&A, 22, 471

Richstone, D., et al. 1998, Nature, 395A, 14

Salucci, P., et al. 2000, MNRAS, 317, 488

Sarzi, M., et al. 2001, ApJ, 550, 65

Sasaki, S. \& Umemura, M. 1996, ApJ, 462, 104

Silk, J., \& Rees, M. 1998, A\&A, 331, L1

Tsuribe, T., \& Umemura, M. 1997, ApJ, 486, 48

Umemura, M., Fukue, J., \& Mineshige, S. 1997, ApJ, 479, L97 1998, MNRAS, 299, 1123

Umemura, M., Loeb, A., \& Turner, E. L. 1993, 419, 459

Wandel, A. 1999, ApJ, 519, L39 